\title{
Stress Distribution in Layered Elastic Creeping Array with a Vertical Cylindrical Shaft
}

\author{
Tatiana Bobyleva ${ }^{1, *}$, Alexei Shamaev ${ }^{2}$ \\ ${ }^{1}$ Moscow state university of civil engineering, Yaroslavskoye shosse, 26, Moscow, Russia, 129337 \\ ${ }^{2}$ Institute for Problems in Mechanics of the Russian Academy of Sciences, 101-1, Pr. Vernadskogo, \\ Moscow, 119526, Russian Federation
}

\begin{abstract}
Construction should be taking into account the influence of time factor on the stability of the structures. In the paper hereditary creep and homogenization theories are used to determine stresses in the layered elastic creeping array with a vertical shaft. Volterra correspondence principle was applied. As a result, the reduction of a time-dependent elastic creeping problem to a corresponding elastic problem became possible. The method proposes a way to determine average (effective) elastic creeping properties and homogenized stress field from known properties of the layers' components. Creep kernels are of a convolution type and are taken in the exponential form. The problem of heterogeneous elastic creeping environment is reduced to a problem of homogeneous transversely isotropic medium. Different boundary conditions on the cylindrical shaft's surface were considered. An analytical solution was obtained. These explicit expressions can be useful for the necessary calculations in the construction practice.
\end{abstract}

\section{Introduction}

Building constructions on the grounds consisting of rocks or inside rocks is an actual problem. Most of the rocks have a heterogeneous structure. Partial differential equations which describing the behavior of such arrays contain rapidly changing coefficients. The gomogenization method is used to solve such problems. This method is developed in the works [1-3]. Examples of its application are given in [4-6]. The problem of a heterogeneous elastic medium is replaced by a problem of a homogeneous medium for which equations have constant coefficients. These coefficients are called effective. Problems for heterogeneous materials with continuously changing characteristics have been solved in [79].

In addition, almost all rocks have the property of creeping, which is the ability to deform with time at constant mechanical stresses [10-11]. Consideration of creeping is of great importance for the stability assessment of the rock massif during construction and exploitation of surface and underground structures. Examples of solving these problems are in the following works [12-16]. A characteristic feature of rheological processes is the dependence of deformation observed at given moment on the character of the whole

*Corresponding author: tatyana2211@,outlook.com 
previous load process. This property of the materials is called hereditary. Hereditary theory is widely used in rock mechanics because it agrees satisfactorily with experience [17-18].

One of the most powerful approaches in solving linear elastic creeping problems consists of the reduction to a corresponding pure elastic problem. Volterra correspondence principle can be applied to the solution of such elastic creeping problems. In the paper at first the Laplace transform was applied to all governing equations describing an elastic creeping array in equilibrium. As a result, all coefficients depend on the transform parameter. Because the array has layered structure, the theory of averaging is applied to the obtained equations. Finally, the equilibrium problem for a transversely isotropic homogeneous elastic array is obtained. The solution of this problem for an array with a vertical cylindrical cavity already exists [19]. We substitute previously obtained averaged (effective) coeficients in this solution and apply an inverse Laplace transform to obtain final solution.

\section{Problem specification and decision}

Let a layered array has pairs of alternating homogeneous layers of two elastic-creeping isotropic materials. These layers are parallel to the horizontal boundary plane. In addition, a vertical shaft extends from this boundary plane. The array and the shaft are assumed semiinfinite. The shaft is bounded by a cylindrical surface of circular cross section of radius $\mathrm{R}$. The problem is to determine stresses in this array in case when the only body force is its weight.

The original constitutive relations are [13]:

$$
-\frac{\partial}{\partial x_{k}}\left(a_{i j k h} \delta(t)+d_{i j k h}\right) * \frac{\partial u_{j}}{\partial x_{h}}=f_{i}(x, t),(i, j, k, h=1 \div 3) .
$$

In (1) the symbol * denotes the convolution operator:

$$
\left(d_{i j k h}(x, t) * \frac{\partial u_{j}}{\partial x_{k}}\right)=\int_{0}^{t} d_{i j k h}(x, t-\tau) \frac{\partial u_{j}(x, \tau)}{\partial x_{k}} d \tau .
$$

$u_{j}(j=1,2,3)$ are components of the displacement, $\delta(t)$ is Dirac-delta function, operation $\left(a_{i j k h} \delta(t)\right) *$ is equivalent to multiplication by $a_{i j k h}$. (Einstein convention for repeated indices is used.) We are considering isotropic materials therefore the tensors in (1) can be expressed in the form $[1-3,13]$ :

$$
\begin{gathered}
a_{i j k h}=\lambda \delta_{i j} \delta_{k h}+\mu\left(\delta_{i k} \delta_{j h}+\delta_{i h} \delta_{j k}\right), \\
d_{i j k h}=-\left(D_{v}(t)-\frac{1}{3} D_{s}(t)\right) \delta_{i j} \delta_{k h}-\frac{1}{2} D_{s}(t)\left(\delta_{i k} \delta_{j h}+\delta_{i h} \delta_{j k}\right) .
\end{gathered}
$$

We denote here by $D_{s}$ and $D_{v}$ the regular part of the shear and the bulk relaxation respectively, by $\delta_{i j}$ Kronecker symbol. Let us assume that the amplitude of the bulk creeping kernel is proportional to the shear amplitude with a coefficient of proportionality $k_{i}$ for each layer that is: $\left(D_{v}\right)_{i}=k_{i}\left(D_{s}\right)_{i}, k_{i}=$ const, $k>0, \quad(i=1,2)$. Further, $D_{s}$ is denoted by $D$. 
A cylindrical coordinate system $(r, \varphi, z)$ with the axis $o z$ directed vertically downward is chosen. Its origin is at the center of the upper circular cross section of the shaft. The thickness ratio of one layer to the cell period $\varepsilon$ is denoted by $h$. Consequently, the ratio of the thickness of the other layer to the cell period will be $1-h . \quad(0<h<1)$. As usual, we denote by $\sigma_{r}, \sigma_{\theta}, \sigma_{z}$ stress components, by $e_{r}, e_{\theta}, e_{z}$ strain components, by $u_{r}, u_{\theta}, u_{z}$ elastic displacements, by $\lambda_{i}, \mu_{i}$ Lame parameters, by $\gamma_{i}$ specific weight, by $D_{i}$ creeping kernel for each layer $(i=1,2)$.

Suppose a surface of the shaft is load-free, namely $\sigma_{r}=0, \sigma_{r z}=0$ at $r=R$. Conditions on a horizontal boundary surface of the rock are: $\sigma_{z}=0, \sigma_{r z}=0$ at $z=0$.

The conditions of ideal contact are taken on the horizontal boundaries of the layers which are: two components of the displacement $u_{r}, u_{z}$ and the normal component of stress parallel the $z$-axis $\sigma_{z}$ are continuous on all contact planes of the layers, i.e. $\left[u_{r}\right]=0,\left[u_{z}\right]=0,\left[\sigma_{z}\right]=0$.

In this problem, all elastic modulus, specific weight and creeping kernels are periodic functions of the coordinate $\xi=\frac{z}{\varepsilon}$ ( $\varepsilon$ is the cell period) and are piecewise constant functions of this variable, i.e., elastic modulus, specific weight and creep kernels have the form [16]:

$$
\begin{gathered}
\lambda_{i}=\left\{\begin{array}{l}
\lambda_{1}, \xi \in[0 ; h], \\
\lambda_{2}, \xi \in[1-h ; 1],
\end{array} \mu_{i}=\left\{\begin{array}{l}
\mu_{1}, \xi \in[0 ; h], \\
\mu_{2}, \xi \in[1-h ; 1],
\end{array} \gamma_{i}=\left\{\begin{array}{l}
\gamma_{1}, \xi \in[0 ; h], \\
\gamma_{2}, \xi \in[1-h ; 1],
\end{array}\right.\right.\right. \\
D_{i}=\left\{\begin{array}{l}
D_{1}, \xi \in[0 ; h], \\
D_{2}, \xi \in[1-h ; 1],
\end{array} \quad 0<h<1, i=1,2 .\right.
\end{gathered}
$$

Let us choose creep kernel of exponential type for each layer: $D_{i}=d_{i} e^{-\alpha_{i} t}$, where $\alpha_{i}, d_{i}$ are constants, $\alpha_{i}>0, d_{i}>0,(i=1,2), t$ is the variable that specifies time. For a long time period such kernel well describes the behaviour of rock massif as shown by the experiments.

We apply the Laplace transform in the time domain to the equations (1)

$$
\tilde{f}(p)=\int_{0}^{\infty} f(t) e^{-p t} d t
$$

The result is the system of elasticity theory with a complex parameter $\mathrm{p}$. We apply homogenization method described in [1-3] to this system. As a result, we have got a transversely isotropic homogeneous medium. The symmetric matrix of its effective coefficients is [16]: 


$$
\begin{aligned}
& \left(\begin{array}{cccccc}
<\tilde{c}_{11}>\quad\left\langle\tilde{c}_{12}>\right. & <\tilde{c}_{13}> & 0 & 0 & 0 \\
<\tilde{c}_{22}> & <\tilde{c}_{23}> & 0 & 0 & 0 \\
& <\tilde{c}_{33}> & 0 & 0 & 0 \\
& & <\tilde{c}_{44}> & 0 & 0 \\
& & & <\tilde{c}_{55}>c & 0 \\
& & & & <\tilde{c}_{66}>
\end{array}\right) \\
& <\tilde{c}_{11}>=<\tilde{c}_{22}>=L-\frac{G_{1} h}{p+\alpha_{1}}-\frac{G_{2}(1-h)}{p+\alpha_{2}}- \\
& -\frac{h(1-h)\left\{\left[\lambda_{2}\left(p+\alpha_{2}\right)-j_{2}\right]\left(p+\alpha_{1}\right)-\left[\lambda_{1}\left(p+\alpha_{1}\right)-j_{1}\right]\left(p+\alpha_{2}\right)\right\}^{2}}{\left\{\left[L_{2}\left(p+\alpha_{2}\right)-G_{2}\right] h\left(p+\alpha_{1}\right)+\left[L_{1}\left(p+\alpha_{1}\right)-G_{1}\right](1-h)\left(p+\alpha_{2}\right)\right\}\left(p+\alpha_{1}\right)\left(p+\alpha_{2}\right)}, \\
& <\tilde{c}_{12}>=l-\frac{j_{1} h}{p+\alpha_{1}}-\frac{j_{2}(1-h)}{p+\alpha_{2}}- \\
& -\frac{h(1-h)\left\{\left[\lambda_{2}\left(p+\alpha_{2}\right)-j_{2}\right]\left(p+\alpha_{1}\right)-\left[\lambda_{1}\left(p+\alpha_{1}\right)-j_{1}\right]\left(p+\alpha_{2}\right)\right\}^{2}}{\left\{\left[L_{2}\left(p+\alpha_{2}\right)-G_{2}\right] h\left(p+\alpha_{1}\right)+\left[L_{1}\left(p+\alpha_{1}\right)-G_{1}\right](1-h)\left(p+\alpha_{2}\right)\right\}\left(p+\alpha_{1}\right)\left(p+\alpha_{2}\right)}, \\
& <\tilde{c}_{13}>=<\tilde{c}_{23}>= \\
& =\frac{\left[\lambda_{1}\left(p+\alpha_{1}\right)-j_{1}\right] h\left[L_{2}\left(p+\alpha_{2}\right)-G_{2}\right]+\left[\lambda_{2}\left(p+\alpha_{2}\right)-j_{2}\right](1-h)\left[L_{1}\left(p+\alpha_{1}\right)-G_{1}\right]}{\left[L_{2}\left(p+\alpha_{2}\right)-G_{2}\right] h\left(p+\alpha_{1}\right)+\left[L_{1}\left(p+\alpha_{1}\right)-G_{1}\right](1-h)\left(p+\alpha_{2}\right)}, \\
& <\tilde{c}_{33}>=\frac{\left[L_{1}\left(p+\alpha_{1}\right)-G_{1}\right]\left[L_{2}\left(p+\alpha_{2}\right)-G_{2}\right]}{\left[L_{2}\left(p+\alpha_{2}\right)-G_{2}\right] h\left(p+\alpha_{1}\right)+\left[L_{1}\left(p+\alpha_{1}\right)-G_{1}\right](1-h)\left(p+\alpha_{2}\right)}, \\
& <\tilde{c}_{44}>=<\tilde{c}_{55}>=\frac{\left[\mu_{1}\left(p+\alpha_{1}\right)-g_{1}\right]\left[\mu_{2}\left(p+\alpha_{2}\right)-g_{2}\right]}{\left[\mu_{2}\left(p+\alpha_{2}\right)-g_{2}\right] h\left(p+\alpha_{1}\right)+\left[\mu_{1}\left(p+\alpha_{1}\right)-g_{1}\right](1-h)\left(p+\alpha_{2}\right)}, \\
& <\tilde{c}_{66}>=M-\frac{g_{1} h}{p+\alpha_{1}}-\frac{g_{2}(1-h)}{p+\alpha_{2}} .
\end{aligned}
$$

In these formulas we have denoted ( $i$ is the layer number):

$$
\begin{aligned}
& L_{i}=\lambda_{i}+2 \mu_{i}, G_{i}=d_{i}\left(k_{i}+\frac{2}{3}\right), j_{i}=d_{i}\left(k_{i}-\frac{1}{3}\right), g_{i}=\frac{d_{i}}{2},(i=1,2), \\
& L=L_{1} h+L_{2}(1-h), l=\lambda_{1} h+\lambda_{2}(1-h), M=\mu_{1} h+\mu_{2}(1-h) .
\end{aligned}
$$

Constitutive stress-strain relations of received transversely isotropic medium look as:

$$
\begin{gathered}
\tilde{\sigma}_{r}=<\tilde{c}_{11}>\tilde{e}_{r}+<\tilde{c}_{12}>\tilde{e}_{\theta}+<\tilde{c}_{13}>\tilde{e}_{z}, \\
\tilde{\sigma}_{\theta}=<\tilde{c}_{12}>\tilde{e}_{r}+<\tilde{c}_{11}>\tilde{e}_{\theta}+<\tilde{c}_{13}>\tilde{e}_{z}, \tilde{\sigma}_{r z}=<\tilde{c}_{44}>\tilde{e}_{r z} .
\end{gathered}
$$


Stress distribution problem for an array of transversely isotropic elastic material with vertical cylindrical cavity was solved in [19]. Tensor of elastic compliances was used in this solution. We reverse (9), so we define the components of deformation via stress components with help of the effective compliance tensor $<\tilde{\mathrm{s}}_{i j}>$ :

$$
\begin{gathered}
\tilde{\varepsilon}_{r}=<\tilde{s}_{11}>\tilde{\sigma}_{r}+<\tilde{s}_{12}>\tilde{\sigma}_{\theta}+<\tilde{s}_{13}>\tilde{\sigma}_{z}, \\
\tilde{\varepsilon}_{\theta}=<\tilde{s}_{12}>\tilde{\sigma}_{r}+<\tilde{s}_{11}>\tilde{\sigma}_{\theta}+<\tilde{s}_{13}>\tilde{\sigma}_{z}, \tilde{\varepsilon}_{r z}=<\tilde{s}_{44}>\tilde{\sigma}_{r z} .
\end{gathered}
$$

Then we find components of this tensor by using (8).

For our problem, stress-strain state of the elastic creeping array can be found by using the aforementioned solution [19] and the received tensor of effective compliances. This is possible because the average environment is transversely isotropic one. The result is:

$$
\begin{gathered}
\tilde{\sigma}_{r}=\frac{<\tilde{s}_{13}>}{<\tilde{s}_{11}>+<\tilde{s}_{12}>}<\gamma>z\left(1-\frac{R^{2}}{r^{2}}\right), \tilde{\sigma}_{\theta}=\frac{<\tilde{s}_{13}>}{<\tilde{s}_{11}>+<\tilde{s}_{12}>}<\gamma>z\left(1+\frac{R^{2}}{r^{2}}\right), \\
\tilde{\sigma}_{z}=-<\gamma>z, \tilde{\sigma}_{r z}=0 .
\end{gathered}
$$

After substitution of the effective compliances we have:

$$
\begin{gathered}
\tilde{\sigma}_{r}=-\left\{\frac{\left[\lambda_{1}\left(p+\alpha_{1}\right)-j_{1}\right] h}{L_{1}\left(p+\alpha_{1}\right)-G_{1}}+\frac{\left[\lambda_{2}\left(p+\alpha_{2}\right)-j_{2}\right](1-h)}{L_{2}\left(p+\alpha_{2}\right)-G_{2}}\right\}\left[\gamma_{1} h+\gamma_{2}(1-h)\right] z\left(1-\frac{R^{2}}{r^{2}}\right), \\
\tilde{\sigma}_{\theta}=-\left\{\frac{\left[\lambda_{1}\left(p+\alpha_{1}\right)-j_{1}\right] h}{L_{1}\left(p+\alpha_{1}\right)-G_{1}}+\frac{\left[\lambda_{2}\left(p+\alpha_{2}\right)-j_{2}\right](1-h)}{L_{2}\left(p+\alpha_{2}\right)-G_{2}}\right\}\left[\gamma_{1} h+\gamma_{2}(1-h)\right] z\left(1+\frac{R^{2}}{r^{2}}\right), \\
\tilde{\sigma}_{z}=-\left[\gamma_{1} h+\gamma_{2}(1-h)\right] z, \tilde{\sigma}_{r z}=0 .
\end{gathered}
$$

Now we use the inverse Laplace transform. As a result, we have stress state of the elastic creeping array with the vertical cylindrical shaft:

$$
\begin{aligned}
& \sigma_{r}=- {\left[e^{-\frac{L_{1} \alpha_{1}-G_{1}}{L_{1}} t} \frac{\left(G_{1} \lambda_{1}-j_{1} L_{1}\right) h}{L_{1}^{2}}+e^{-\frac{L_{2} \alpha_{2}-G_{2}}{L_{2}} t} \frac{\left(G_{2} \lambda_{2}-j_{2} L_{2}\right)(1-h)}{L_{2}^{2}}+\right.} \\
&\left.+\frac{\lambda_{1} L_{2} h+\lambda_{2} L_{1}(1-h)}{L_{1} L_{2}} \delta(t)\right]\left[\gamma_{1} h+\gamma_{2}(1-h)\right] z\left(1-\frac{R^{2}}{r^{2}}\right), \\
& \sigma_{\theta}=- {\left[e^{-\frac{L_{1} \alpha_{1}-G_{1}}{L_{1}} t} \frac{\left(G_{1} \lambda_{1}-j_{1} L_{1}\right) h}{L_{1}^{2}}+e^{-\frac{L_{2} \alpha_{2}-G_{2}}{L_{2}} t} \frac{\left(G_{2} \lambda_{2}-j_{2} L_{2}\right)(1-h)}{L_{2}^{2}}+\right.} \\
&+\left.\frac{\lambda_{1} L_{2} h+\lambda_{2} L_{1}(1-h)}{L_{1} L_{2}} \delta(t)\right]\left[\gamma_{1} h+\gamma_{2}(1-h)\right] z\left(1+\frac{R^{2}}{r^{2}}\right), \\
& \sigma_{z}=-\left[\gamma_{1} h+\gamma_{2}(1-h)\right] z, \sigma_{r z}=0 .
\end{aligned}
$$


Laplace transforms of radial and axial displacements $u_{r}, u_{z}$ in this case can be found by the formulas:

$$
\begin{gathered}
\tilde{u}_{r}=\frac{\left(<\tilde{s}_{11}>-<\tilde{s}_{12}>\right)<\tilde{s}_{13}>}{<\tilde{s}_{11}>+<\tilde{s}_{12}>}<\gamma>z \frac{R^{2}}{r}, \\
\tilde{u}_{z}=\frac{2<\tilde{s}_{13}>^{2}-<\tilde{s}_{33}>\left(<\tilde{s}_{11}>+<\tilde{s}_{12}>\right)}{2\left(<\tilde{s}_{11}>+<\tilde{s}_{12}>\right)}<\gamma>z^{2}- \\
-\frac{\left(<\tilde{s}_{11}>-<\tilde{s}_{12}>\right)<\tilde{s}_{13}>}{<\tilde{s}_{11}>+<\tilde{s}_{12}>}<\gamma>R^{2} \ln r+C,
\end{gathered}
$$

where $\mathrm{C}$ is arbitrary constant.

After substitution of the effective compliances these formulas take the form of:

$$
\begin{gathered}
\tilde{u}_{r}=\left\{\sum_{i=1}^{2} \frac{\left[j_{i}-\lambda_{i}\left(p+\alpha_{i}\right)\right] H_{i}}{\left[L_{i}\left(p+\alpha_{i}\right)-G_{i}\right] A}\right\}\left(p+\alpha_{1}\right)\left(p+\alpha_{2}\right)\left[h \gamma_{1}+(1-h) \gamma_{2}\right] z \frac{R^{2}}{r}, \\
\tilde{u}_{z}=-\left\{\sum_{i=1}^{n} \frac{\left(p+\alpha_{i}\right) H_{i}}{2\left[L_{i}\left(p+\alpha_{i}\right)-G_{i}\right]}\right\}\left[h \gamma_{1}+(1-h) \gamma_{2}\right] z^{2}+ \\
+\left\{\sum_{i=1}^{2} \frac{\left[\lambda_{i}\left(p+\alpha_{i}\right)-j_{i}\right] H_{i}}{\left[L_{i}\left(p+\alpha_{i}\right)-G_{i}\right] A}\right\}\left(p+\alpha_{1}\right)\left(p+\alpha_{2}\right)\left[h \gamma_{1}+(1-h) \gamma_{2}\right] R^{2} \ln r+C,
\end{gathered}
$$

We denoted by $A=2 M\left(p+\alpha_{1}\right)\left(p+\alpha_{2}\right)-d_{1} h\left(p+\alpha_{2}\right)-d_{2}(1-h)\left(p+\alpha_{1}\right)$ and by

$$
H_{i}=\left\{\begin{array}{l}
h, \text { if } i=1 \\
1-h, \text { if } i=2 .
\end{array}\right.
$$

After applying the inverse Laplace transform we have :

$$
\begin{gathered}
u_{r}=\frac{1}{2 M} \sum_{i=1}^{2} H_{i}\left\{\frac{e^{-\frac{L_{i} \alpha_{i}-G_{i}}{L_{i}} t} G_{i}\left(j_{i} L_{i}-G_{i} \lambda_{i}\right)\left[G_{i}+(-1)^{i} L_{i}\left(\alpha_{1}-\alpha_{2}\right)\right]}{L_{i}^{2}\left[L_{i}\left(m_{1}+\alpha_{i}\right)-G_{i}\right]\left[L_{i}\left(m_{2}+\alpha_{i}\right)-G_{i}\right]}+\right. \\
\left.+\sum_{n=1}^{2} \frac{(-1)^{n} e^{m_{n} t}\left(m_{n}+\alpha_{1}\right)\left(m_{n}+\alpha_{2}\right)\left[\lambda_{i}\left(m_{n}+\alpha_{i}\right)-j_{i}\right]}{\left(m_{1}-m_{2}\right)\left[L_{i}\left(m_{n}+\alpha_{i}\right)-G_{i}\right]}-\frac{\lambda_{i}}{L_{i}} \delta(t)\right\}\left[h \gamma_{1}+(1-h) \gamma_{2}\right] z \frac{R^{2}}{r}, \\
u_{z}=\frac{\left[h \gamma_{1}+(1-h) \gamma_{2}\right]}{2}\left\{-\left[\sum_{i=1}^{2} \frac{e^{-\frac{L_{i} \alpha_{i}-G_{i}}{L_{i}} t} H_{i} G_{i}}{L_{i}^{2}}+\frac{L_{1}(1-h)+L_{2} h}{L_{1} L_{2}} \delta(t)\right] z^{2}+\right.
\end{gathered}
$$




$$
\begin{aligned}
& +\frac{1}{M} \sum_{i=1}^{2} H_{i}\left[\frac{e^{-\frac{L_{i} \alpha_{i}-G_{i}}{L_{i}} t} G_{i}\left(G_{i} \lambda_{i}-j_{i} L_{i}\right)\left[G_{i}+(-1)^{i} L_{i}\left(\alpha_{1}-\alpha_{2}\right)\right]}{L_{i}^{2}\left[L_{i}\left(m_{1}+\alpha_{i}\right)-G_{i}\right]\left[L_{i}\left(m_{2}+\alpha_{i}\right)-G_{i}\right]}+\right. \\
& \left.\left.+\sum_{n=1}^{2} \frac{(-1)^{n+1} e^{m_{n} t}\left(m_{n}+\alpha_{1}\right)\left(m_{n}+\alpha_{2}\right)\left[\lambda_{i}\left(m_{n}+\alpha_{i}\right)-j_{i}\right]}{\left(m_{1}-m_{2}\right)\left[L_{i}\left(m_{n}+\alpha_{i}\right)-G_{i}\right]}+\frac{\lambda_{i}}{L_{i}} \delta(t)\right] R^{2} \ln r\right\}+C .
\end{aligned}
$$

In formulas (16) $m_{1}, m_{2}$ are the roots of the following quadratic equation:

$$
2 M\left(p+\alpha_{1}\right)\left(p+\alpha_{2}\right)-d_{1} h\left(p+\alpha_{2}\right)-d_{2}(1-h)\left(p+\alpha_{1}\right)=0,
$$

that has a positive discriminant $D=\left[d_{1} h+d_{2}(1-h)+2 M\left(\alpha_{2}-\alpha_{1}\right)\right]^{2}+4 d_{1} d_{2} h(1-h)$, because $d_{1}>0, d_{2}>0,0<h<1$. Therefore, $m_{1}, m_{2}$ there will always be real numbers.

Let's consider also the case when the shaft is filled with liquid to the brim. Pressure of this liquid on to the surface of the shaft is equal to $q z$. Stress components in this case are the following:

$$
\begin{aligned}
\sigma_{r} & =-\left[e^{-\frac{L_{1} \alpha_{1}-G_{1}}{L_{1}} t} \frac{\left(G_{1} \lambda_{1}-j_{1} L_{1}\right) h}{L_{1}^{2}}+e^{-\frac{L_{2} \alpha_{2}-G_{2}}{L_{2}} t} \frac{\left(G_{2} \lambda_{2}-j_{2} L_{2}\right)(1-h)}{L_{2}^{2}}+\right. \\
& \left.+\frac{\lambda_{1} L_{1} h+\lambda_{2} L_{2}(1-h)}{L_{1} L_{2}} \delta(t)\right]\left[\gamma_{1} h+\gamma_{2}(1-h)\right] z\left(1-\frac{R^{2}}{r^{2}}\right)-\frac{q R^{2}}{r^{2}} z, \\
\sigma_{\theta}= & -\left[e^{-\frac{L_{1} \alpha_{1}-G_{1}}{L_{1}} t} \frac{\left(G_{1} \lambda_{1}-j_{1} L_{1}\right) h}{L_{1}^{2}}+e^{-\frac{L_{2} \alpha_{2}-G_{2}}{L_{2}} t} \frac{\left(G_{2} \lambda_{2}-j_{2} L_{2}\right)(1-h)}{L_{2}^{2}}+\right. \\
+ & \left.\frac{\lambda_{1} L_{1} h+\lambda_{2} L_{2}(1-h)}{L_{1} L_{2}} \delta(t)\right]\left[\gamma_{1} h+\gamma_{2}(1-h)\right] z\left(1+\frac{R^{2}}{r^{2}}\right)+\frac{q R^{2}}{r^{2}} z,
\end{aligned}
$$

Now let's assume that the surface of the shaft is rigidly fixed, for example, with a pipe. In this case, boundary conditions on this surface are the following: $u_{r}=0, \sigma_{r z}=0$ if $r=R$. In this case, shaft has no effect on stress components they will be the same as in the rock without shaft. Array will exert the following pressure on the pipe's wall

$$
\sigma_{r}=-\left[\sum_{i=1}^{2} e^{-\frac{L_{i} \alpha_{i}-G_{i}}{L_{i}} t} \frac{\left(G_{i} \lambda_{i}-j_{i} L_{i}\right) H_{i}}{L_{i}^{2}}+\frac{\lambda_{1} L_{2} h+\lambda_{2} L_{1}(1-h)}{L_{1} L_{2}} \delta(t)\right]\left[\gamma_{1} h+\gamma_{2}(1-h)\right] z
$$

Stress distribution will be the same as in the array without shaft. 


\section{Conclusion}

The article presents a method of calculating stress-strain state of layered elastic creeping array with a vertically shaft. The theory of homogenization and the creeping theory were applied to solve this problem. Prediction of subsidence of the earth's surface is possible on the basis of the obtained solution. A function of creep has a form of a spectral function of two parameters. Method, proposed in this paper, allows to reduce the solution of the elastic creep problem of the heterogeneous array to the problem of pure elasticity for a transversely isotropic medium.

As a result, obtained formulas give an exact analytical solution for determination the stress-strain state of elastic creeping layered array with a vertical shaft. This exact solution can be used in construction.

\section{References}

1. O.A. Oleynik, A.S. Shamaev, G.A. Yosifian, Mathematical Problems in Elasticity and Homogenization (Elsevier, North-Holland, 1992)

2. D.I. Bardzokas, A.I. Zobnin, Mathematical Modelling of Physical Processes in Composite Materials of Periodical Structures (URSS, Moscow, 2003)

3. E. Sanchez-Palencia, Non-Homogeneous Media and Vibration Theory (Springer, Berlin, 1980)

4. A.S. Shamaev, V.V. Shumilova, Doklady Physics 56 (1), 43 (2011)

5. T. Bobyleva, Procedia Engineering 153, 103 (2016)

6. T.N. Bobyleva, Scientific review 24, 18 (2016)

7. V.I. Andreev, Some problems and methods of mechanics inhomogeneous bodies (ASV Publ. House, Moscow, 2002)

8. V.I. Andreev, D.A. Kapliy, Procedia Engineering 111, 36 (2015)

9. N.Yu.Tsybina, R.A.Turusova, V.I. Andreev, Procedia Engineering 153, 51 (2016)

10. Yu.N. Rabotnov, Creep of Structural Elements (Nauka, Moscow, 1966)

11. A.A. Ilyushin, B.E. Pobedrya, Foundations of the mathematical theory of thermoviscoelasticity (Nauka, Moscow, 1970)

12. A.S. Shamaev, V.V. Shumilova, Proceedings of the Steklov Institute of Mathematics 295, 218 (2016)

13. A.S. Shamaev, V.V. Shumilova, Proceedings of the Steklov Institute of Mathematics 295, 229 (2016)

14. Z.G. Ter-Martirosyan, A.Z. Ter-Martirosyan, Soil Mechanics and Foundation Engineering 49 (6), 219 (2013)

15. Z.G. Ter-Martirosyan, M.V. Proshin, Soil Mechanics and Foundation Engineering 43 (2), 56 (2006)

16. T.N. Bobyleva, MATEC Web of Conferences 86, 01024 (2016)

17. S.A. Konstantinova, V.M. Pestrenin, I.V. Pestrenina, Proceedings of higher educational institutions. Mining journal 4, 92 (2007)

18. M. H. Gabdrahimov, M.N. Dedyukin, Research on mining. The proceedings of the conference on the results of scientific research (Perm Polytechnic Institute, Perm, 1968)

19. S.G. Lehnickii, Theory of the Elasticity of the Anisotropic Body (Nauka, Moscow, 1977) 